\title{
A Boosting Frame Work for Improved Content Based Image Retrieval
}

\author{
Sasi Kumar M. ${ }^{1 *}$ and Y. S. Kumaraswamy ${ }^{2}$ \\ 1Research Scholar, Department of CSE, Sathyabama University, Chennai, India; msksasi@yahoo.com \\ 2 Sr. Professor \& HOD, Dept. of MCA, Dayananda Sagar College of Engineering, \\ Bangalore, India; yskldswamy@yahoo.co.in
}

\begin{abstract}
This paper deals with medical image retrieval for retrieving images similar to query images from a database. Retrieval of archived digital medical images is always a challenge that is still being researched all the more so as such images are of paramount importance in patient diagnosis, therapy, surgical planning, medical reference, and medical training. This paper proposes using the Discrete Sine Transform (DST) for relevant feature extraction, and applies Boosting classification techniques to locate the relevant images. In this study, the boosting is used with J48 and decision stump. Experimental results show that the classification accuracy achieved is fairly good.
\end{abstract}

Keywords: Content Based Image Retrieval (CBIR), Medical Images, Discrete Sine Transform (DST), Boosting, J48, Decision Stump.

\section{Introduction}

Visual information from the digital images such as MRI, CT is widely used in medical applications. Management of image database in the early 80's was based on image textual annotation $[1,2]$. They were manually annotated with keywords necessitating a lot of labour and images were retrieved through semantic questions. The huge volume of images generated at present has made image annotation both impractical and impossible thereby necessitating and automatic classification system for image retrieval. Formerly, image retrieval based on visual features with text annotation was used $[3,4,5]$. In the present scenario, to handle huge amount of medical image data, image retrieval based on visual information is crucial in medical applications [6]. An efficient retrieval system should:

- Extract information from images to a multi-dimensional feature vector

\footnotetext{
* Corresponding author:
}

Sasi Kumar. M (msksasi@yahoo.com)
- Compute distance metrics in a quantifiable manner, and

- Identify database images with lowest distance metrics from query images

Database images are pre-processed automatically to extract features, generating feature vectors in content based image retrieval (CBIR) system. Feature vectors are stored in feature database and images classified. The query image is also pre-processed likewise to extract features and on the basis of this similarity, appropriate database images are retrieved. Thus image retrieval has necessity to play a major role in handling huge amounts of visual information in medical applications. [7]. Image retrieval system performance is dependent on a multi-dimensional feature vector formed using extracted image information, computing of similarity measures and proper and correct identification of database images with lowest distance metrics in relation to query image. Transform methods are usually resorted to image 
processing as many coefficients can be ignored in reducing feature vector size.

Low level features are popular in Image Retrieval systems $[8,9]$, as every model combines low-level features to define a distance metric that quantifies similarities between image models. One shortcoming of this procedure is that low-level image features may not always capture a human perception of image similarity. To state otherwise, semantic image content is hard for feature extraction with low level image features alone and this is called the semantic gap problem [10]. Medical Image Retrieval systems are not the same as regular image retrieval systems. For one, retrieval is linked to pathological conditions which are highly local and so retrieval based on global signatures would be useless for medical databases. Converting images from the spatial to a frequency domain is a widely used image retrieval procedure available in the literature $[11,12,13]$.

This paper proposes frequency vector extraction from medical images through Discrete Sine Transform (DST) with the application of Boosting classification techniques. This paper is organized as follows: Section 2 reviews related works available in the literature. Section 3 describes Discrete Sine Transform through a down-sampling technique, and the classifier methodology, Section 4 details the experiment and results obtained. Section 5 concludes the paper with a discussion on the obtained results.

\section{Related Works}

Rajkumar, and Rajkumar, [14] presented a 2 step medical image retrieval framework for retrieval of similar images from various features. A subset of images was selected using a wavelet filtering process and the image was decomposed into 6 levels using wavelet transforms with their energies being extracted. Euclidean distance was used to match similar query and database image and dimensions were reduced through use of PCA. Finally, calculated eigen vectors and similarity measures were applied to ensure efficient medical image retrieval. There was improved retrieval accuracy because of reduced search space efficiency. Experiments with 200 medical images proved the accuracy of the proposed method with regard to precision and recall rate.

Kak, and Pavlopoulou, 2002 [15] investigated problems unique to automated image retrieval from big medical databases with HRCT lung and liver images being the dataset. A feature selection scatter-shot approach is employed to obtain an exhaustive set of low-level features. A customized queries approach (CQA) found features that discriminated major classes, and then best ' $n$ ' images are obtained by customizing the query. The system improved overall diagnosis accuracy from $32.5 \%$ to $64.6 \%$.

In photo image management, the most significant operation is the detection of image orientation automatically. Lei Zhang et al., [16] proposed an automated technique for the purpose of evaluation of image orientations. Based on the confidence score of the orientation detection, the proposed technique has the ability to reject images. Further, the classification of images into indoor and outdoor is done and then further refining of the orientation detection is performed using the obtained classification result. The features are combined by subtraction operation and the essential features are chosen by implementing boosting algorithm in order to choose the features most sensitive to the rotation. The proposed method possess many benefits, they are: faster classification speed, minute size of the model and beneficial rejection approach.

Tieu, and Viola [17] introduced a scheme using a very large number of highly selective features and well-organized learning of queries for the purpose of image retrieval. On the assumption that the generation of every image is by a sparse set of visual "causes" and the images that are visually same share "causes" is the predication of the proposed scheme. Some exemplar images and the AdaBoost algorithm are chosen at query time by a user in order to study a classification function that relies on a few of the generally suitable aspects. A more efficient classification function is yielded. A natural mechanism is provided by the AdaBoost framework for the purpose of integrating relevance feedback additionally.

Hertz et al., [18] trained binary classifiers with margins in order to learn distance functions in which the classifiers are defined on the product space of pairs of images. The classifiers are trained to distinguish between pairs that comprises images are from the equivalent class and pairs including images from various classes. A distance function used is the signed margin. Many variants regarding this idea are explored on the basis of implementing the product space classifiers, SVM and Boosting algorithms. A distance learning technique is the major contribution of this work that is a combination of boosting hypotheses on the product space including a weak learner based on partitioning the original feature space. The proposed scheme facilitates the integration of unlabeled data into the training process. The results obtained from two cases show that the proposed GMM based boosting method shows high-throughput and its generalization to unseen classes is superior to all the other techniques. 


\section{Methodology}

\subsection{Discrete Sine Transform (DST)}

In this study, the feature vector from each image was extracted using the Discrete Sine Transform (DST). DST extracts feature vectors from every image and pixels one length away from each other are selected. The pseudo algorithm is shown below:

1. Compute Image size $M \times N$

2. For each alternate value ' $i$ ' in array $M$ and array size less than $\mathrm{M}$ or $\mathrm{M}+1$

3. For each alternate value ' $j$ ' in array $N$ and array size less than $\mathrm{N}$ or $\mathrm{N}+1$

4. Compute DST (array $\left.\left[x_{i}, y_{j}\right]\right)$

5. Store computed value in one dimensional array

6. Repeat from step 1 till all images are computed

The DST and discrete Fourier transform (DFT) are similar the only difference being in the use of numbers. The discrete sine transform is represented by:

$S_{k}=p_{K} \sum_{n=0}^{N-1} x_{n} \sin \frac{\pi\left(n+\frac{1}{2}\right)(k+1)}{N} k=0,1,2, \ldots \ldots \ldots . ., N-1$

$p_{k}=\sqrt{\frac{2-\delta_{k, 0}}{N}}$

where $\mathrm{x}_{\mathrm{n}}$ is the original vector on $\mathrm{N}$ real numbers. $\delta$ is the Kronecker delta. DST operates on real data with odd symmetry and hence output data is shifted by half a sample. The inverse of DST is given by:

$S_{k}^{I I I}=p_{K} \sum_{n=0}^{N-1} x_{n} q_{n} \sin \frac{\pi(n+1)\left(k+\frac{1}{2}\right)}{N} k=0,1,2, \ldots \ldots \ldots . ., N-1$

$p=\sqrt{\frac{2}{N}}$

$q_{n}=\sqrt{\frac{1}{1+\delta_{n, 0}}}$

\subsection{Bagging}

For enhancing the accuracy of any given learning algorithm, 'Boosting' method is used. Boosting is a machine learning method which finds many rough rules and combines it to produce accurate classification [19]. The boosting algorithm finds rules repeatedly using different subset of the training set on the base learning algorithm $[21,22,23]$. AdaBoost
[20] is the most commonly used boosting technique. For a training set $\left(x_{1}, y_{1}\right) \ldots . . .,\left(x_{m}, y_{m}\right)$ where $x_{i} \in X, y_{i} \in Y=\{-1,1\}$.

The pseudocode for AdaBoost is as follows [19]:

1. Initialize $D_{1}(i)=1 / m$

2. For number of iteration $t=1, \ldots, T$

3. Train base learner using distribution $D_{t}$

4. Get base classifier $h_{t}: X \rightarrow \Re$

5. Choose $\alpha_{t} \in \mathfrak{R}$

6. Update

$$
D_{t+1}(i)=\frac{D_{t}(i) \exp \left(-a y_{i} h_{t}\left(x_{i}\right)\right)}{Z_{t}}
$$

7. Output of final classifier:

$$
H(x)=\operatorname{sign}\left(\sum_{t=1}^{T} a_{t} h_{t}(x)\right)
$$

In this study, the boosting is used with $\mathrm{J} 48$ and decision stump.

\section{Experimental Setup and Results}

A program was developed in LabVIEW which handles multiple input images, outputting the co-efficient as a comma separated values with down-sampling. Various MRI scan images and noisy images forms the dataset used for evaluation. Sample images from the dataset are seen in Figure 1. Many of them were rotated by a 90 degree angle to simulate real time database search. Four medical image types were used in the experiment with different noise degrees. 57 MRI scan images were used as inputs and classified using boosting with J48 and boosting with decision stump. Classification was undertaken with $60 \%$ of data as training set with the remaining being a test set.

The results and classification accuracy and Root Mean Squared Error is tabulated in Table 1, Figure 2 and Figure 3.

It is evident from the graphs that the Boosting techniques achieve better classification accuracy than bagging and Boosting with J48 performance is better than the other techniques.

\section{Conclusion}

This paper investigates medical image retrieval from a database. Discrete Sine Transform (DST) is used for feature extraction and Boosting classification techniques finds 

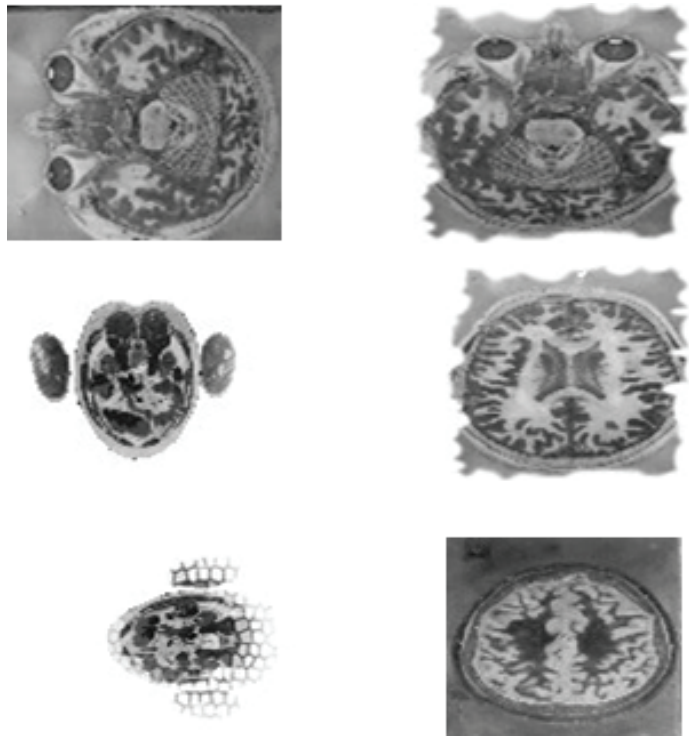

Figure 1. Sample images used in the medical retrieval system.

Table 1. Experimental results for various techniques

\begin{tabular}{lcc}
\hline Technique Used & $\begin{array}{c}\text { Classification } \\
\text { Accuracy \% }\end{array}$ & RMSE \\
\hline Bagging with J48 algorithm & 84.21 & 0.2338 \\
Bagging with decision stump & 80.7 & 0.3347 \\
Boosting with J48 algorithm & $\mathbf{8 5 . 9 6}$ & 0.1942 \\
Boosting with decision stump & 84.21 & 0.2926 \\
\hline
\end{tabular}

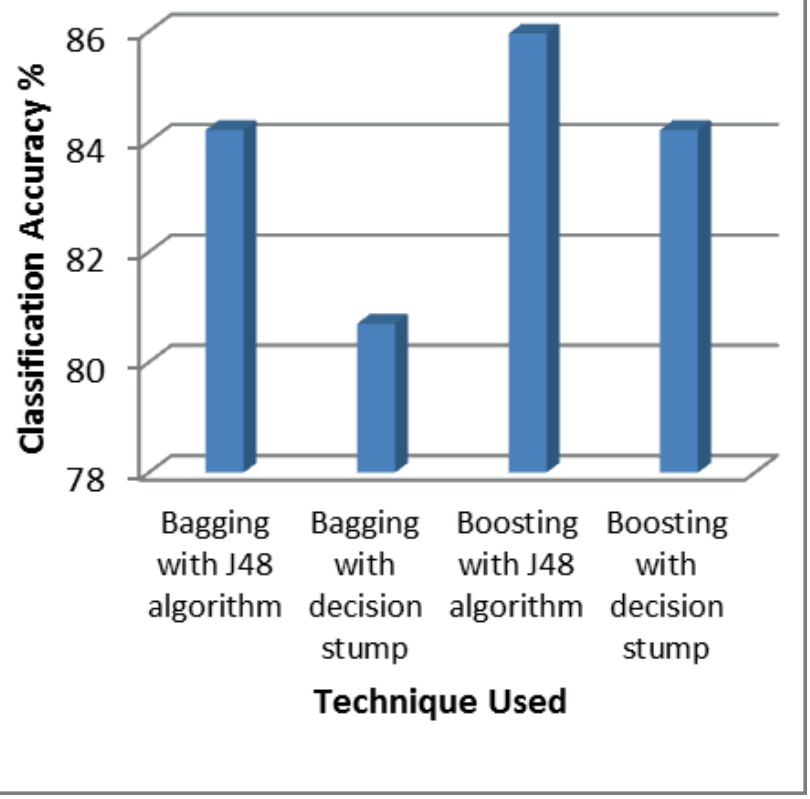

Figure 2. Classification Accuracy.

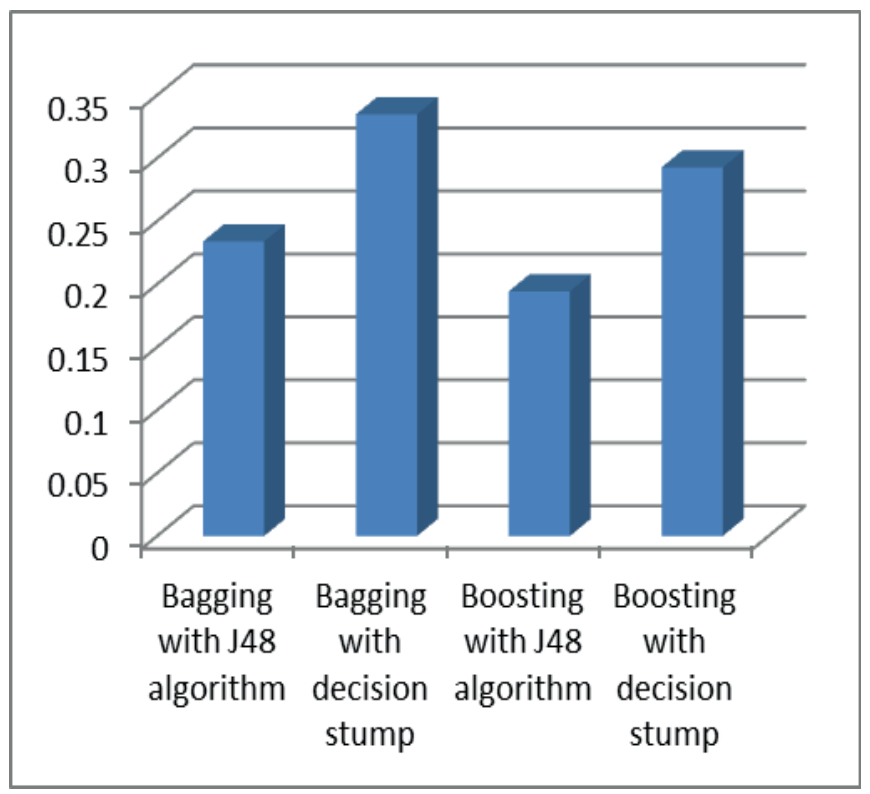

Figure 3. Root Mean Squared Error.

relevant images. Boosting is done with J48 and with decision stump. Classification accuracy results are fairly good. Boosting techniques achieve better classification accuracy than Bagging. Boosting with J48 performance is better than the other techniques achieving accuracy of $85.96 \%$.

\section{References}

1. Chang N S, and Fu K S (1980). Query by pictorial example, IEEE Transactions on Software Engineering, vol 6(6), 519-524.

2. Chang S K, Yan C W et al. (1988). An intelligent image database system, IEEE Transactions on Software Engineering, vol 14(5), 681-688.

3. Dowe J (1993). Content-based retrieval in multimedia imaging, Proceeding SPIE Storage and Retrieval for Image and Video Database, vol 1908.

4. Faloutsos C et al. (1994). Efficient and effective querying by image content, Journal of intelligent information systems, vol 3(3-4), 231-262.

5. Bhadoria S, and Dethe C G (2010). Study of medical image retrieval system, 2010 International Conference on Data Storage and Data Engineering, 192-196.

6. Müller H, Michoux N, et al. (2004). A review of content based image retrieval systems in medical applications-clinical benefits and future directions, International Journal of Medical Informatics, vol 73(1), 1-23.

7. Kumar M S, and Kumaraswamy Y S (2011). Medical image retrieval system using an improved MLP neural network, European Journal of Scientific Research, vol 66(4), 532-540. 
8. Manjunath B S, Ohm J R et al. (2001). Color and texture descriptors, IEEE Transactions on Circuits and Systems for Video Technology, vol 11(6), 703-715.

9. Jing F, Li M et al. (2004). An efficient and effective region-based image retrieval framework, IEEE Transactions on Image Processing, vol 13(5), 699-709.

10. Rui Y, Huang T S et al. (1999). Image retrieval: Current techniques, promising directions, and open issues, Journal of Visual Communication and Image Representation, vol 10(1), 39-62.

11. Kelly P, Cannon T et al. (1995). Query by image example: the comparison algorithm for navigating digital image databases (CANDID) approach. Storage and Retrieval for Image and Video Databases III, vol 2420, 238-248.

12. Kekre H B, and Mishra D (2010). digital image search \& retrieval using FFT sectors of color images, International Journal of Computer Science and Engineering (IJCSE), vol $2(2), 368-372$.

13. Iakovidis D K, Pelekis N, et al. (2009). A pattern similarity scheme for medical image retrieval, IEEE Transactions on Information Technology in Biomedicine, vol 13(4), $442-450$.

14. Rajakumar K, and Rajakumar K (2011). An integrated approach for medical image retrieval using PCA and energy efficient wavelet transform, European Journal of Scientific Research, vol 51(3), 340-348.

15. Kak A, and Pavlopoulou C (2002). Content-based image retrieval from large medical databases, Proceedings. First International Symposium on 3D Data Processing Visualization and Transmission, 2002, 138-147.
16. Zhang L, Li M, et al. (2002). Boosting image orientation detection with indoor vs. outdoor classification, Proceedings Sixth IEEE Workshop on Applications of Computer Vision, 2002. (WACV 2002), 95-99.

17. Tieu K, and Viola P (2000). Boosting image retrieval, Proceedings of the IEEE Conference on Computer Vision and Pattern Recognition, vol 1, 228-235.

18. Hertz T, Bar-Hillel A et al. (2004). Learning distance functions for image retrieval, Proceedings of the 2004 IEEE Computer Society Conference on Computer Vision and Pattern Recognition, CVPR 2004, vol 2, II-570-II-577.

19. Liu Y, Rong J et al. (2010). A boosting framework for visuality-preserving distance metric learning and its application to medical image retrieval pattern analysis and machine intelligence, IEEE Transactions on Pattern Analysis and Machine Intelligence, vol 32(1), 30-44.

20. Freund Y, and Schapire R E (1997). A decision-theoretic generalization of on-line learning and an application to boosting, Journal of Computer and System Sciences, vol 55(1), 119-139.

21. Schapire R (2002). The boosting approach to machine learning An overview, MSRI Workshop on Nonlinear Estimation and Classification.

22. Quellec G (2010). Medical Case Retrieval From a Committee of Decision Trees IEEE Transactions on Information Technology in Biomedicine, vol 14(5), 1227-1235.

23. Freund Y, and Schapire R E (1997). A decision-theoretic generalization of on-line learning and an application to boosting, Journal of Computer and System Sciences, vol 55(1), 119-139. 